\title{
Prevalence and Risk Factors of Infection in the Representative COVID-19 Cohort Munich
}

\author{
Michael Pritsch 1,2,+(D), Katja Radon ${ }^{3,4,5,+}$, Abhishek Bakuli 1,+(D), Ronan Le Gleut $6,7,+$, Laura Olbrich 1,2 ${ }^{\mathbb{D}}$, \\ Jessica Michelle Guggenbüehl Noller ${ }^{1}$, Elmar Saathoff ${ }^{1,2}$, Noemi Castelletti ${ }^{1}$, Mercè Garí ${ }^{6} \mathbb{D}$, Peter Pütz $^{6,8}$, \\ Yannik Schälte ${ }^{6,9}$, Turid Frahnow ${ }^{6,8} \mathbb{D}$, Roman Wölfel ${ }^{2,10}$, Camilla Rothe ${ }^{1}$, Michel Pletschette ${ }^{1}$, Dafni Metaxa ${ }^{1}$, \\ Felix Forster ${ }^{3,5}$, Verena Thiel ${ }^{1}$, Friedrich Rieß ${ }^{1,2}$, Maximilian Nikolaus Diefenbach ${ }^{1}$, Günter Fröschl ${ }^{1,4} \mathbb{D}_{\text {, }}$ \\ Jan Bruger ${ }^{1}$, Simon Winter ${ }^{1}$, Jonathan Frese ${ }^{1}$, Kerstin Puchinger ${ }^{1}$, Isabel Brand ${ }^{1}$, Inge Kroidl ${ }^{1,2}$, \\ Jan Hasenauer $6,9,11,+$, Christiane Fuchs $6,7,8,9,+(\mathbb{D}$, Andreas Wieser 1,2,+, Michael Hoelscher 1,2,4,*,+ \\ and on behalf of the KoCo19 study group $\ddagger$
}

1 Division of Infectious Diseases and Tropical Medicine, University Hospital, LMU Munich, 80802 Munich, Germany; pritsch@lrz.uni-muenchen.de (M.P.); bakuli@lrz.uni-muenchen.de (A.B.); olbrich@lrz.uni-muenchen.de (L.O.); guggenbuehl@lrz.uni-muenchen.de (J.M.G.N.); saathoff@lrz.uni-muenchen.de (E.S.); castelletti@lrz.uni-muenchen.de (N.C.);

check for

updates

Citation: Pritsch, M.; Radon, K.; Bakuli, A.; Le Gleut, R.; Olbrich, L.; Guggenbüehl Noller, J.M.; Saathoff, E.; Castelletti, N.; Garí, M.; Pütz, P.; et al. Prevalence and Risk Factors of Infection in the Representative COVID-19 Cohort Munich. Int. J. Environ. Res. Public Health 2021, 18, 3572. https://doi.org/10.3390/ ijerph18073572

Academic Editors:

Jadwiga Wojkowska-Mach,

Maria Luisa Moro,

Monika Chlebowicz, Anna Różańska and Paul B. Tchounwou

Received: 18 February 2021

Accepted: 27 March 2021

Published: 30 March 2021

Publisher's Note: MDPI stays neutral with regard to jurisdictional claims in published maps and institutional affiliations.

Copyright: (c) 2021 by the authors. Licensee MDPI, Basel, Switzerland. This article is an open access article distributed under the terms and conditions of the Creative Commons Attribution (CC BY) license (https:// creativecommons.org/licenses/by/ $4.0 /)$ rothe@lrz.uni-muenchen.de (C.R.); Michel.Pletschette@lrz.uni-muenchen.de (M.P.); metaxa@lrz.uni-muenchen.de (D.M.); Verena.Thiel@lrz.uni-muenchen.de (V.T.); Friedrich.Riess@lrz.uni-muenchen.de (F.R.); diefenbach@1rz.uni-muenchen.de (M.N.D.); froeschl@lrz.uni-muenchen.de (G.F.); jan.bruger@med.uni-muenchen.de (J.B.); simon.winter@med.uni-muenchen.de (S.W.); jonathan.frese@med.uni-muenchen.de (J.F.); kerstin.puchinger@med.uni-muenchen.de (K.P.); isabel.brand@med.uni-muenchen.de (I.B.); ikroidl@lrz.uni-muenchen.de (I.K.); wieser@mvp.lmu.de (A.W.)

2 German Center for Infection Research (DZIF), Partner Site Munich, 80802 Munich, Germany; RomanWoelfel@Bundeswehr.org

3 Institute and Outpatient Clinic for Occupational, Social and Environmental Medicine, University Hospital, LMU Munich, 80336 Munich, Germany; katja.radon@med.uni-muenchen.de (K.R.);

Felix.Forster@med.uni-muenchen.de (F.F.)

4 Center for International Health (CIH), University Hospital, LMU Munich, 80336 Munich, Germany

5 Comprehensive Pneumology Center (CPC) Munich, German Center for Lung Research (DZL), 89337 Munich, Germany

6 Helmholtz Zentrum München-German Research Center for Environmental Health, Institute of Computational Biology, 85764 Neuherberg, Germany; ronan.legleut@helmholtz-muenchen.de (R.L.G.); merce.gari@helmholtz-muenchen.de (M.G.); peter.puetz@uni-bielefeld.de (P.P.); yannik.schaelte@helmholtz-muenchen.de (Y.S.); turid.frahnow@helmholtz-muenchen.de (T.F.); jan.hasenauer@uni-bonn.de (J.H.); christiane.fuchs@helmholtz-muenchen.de (C.F.)

7 Helmholtz Zentrum München-German Research Center for Environmental Health, Core Facility Statistical Consulting, 85764 Neuherberg, Germany

8 Faculty of Business Administration and Economics, Bielefeld University, 33615 Bielefeld, Germany

9 Center for Mathematics, Technische Universität München, 85748 Garching, Germany

10 Bundeswehr Institute of Microbiology, 80937 Munich, Germany

11 Interdisciplinary Research Unit Mathematics and Life Sciences, University of Bonn, 53113 Bonn, Germany

*orrespondence: hoelscher@lrz.uni-muenchen.de; Tel.: +49-89-44005-9801

$+\quad$ Those authors contributed equally.

$\ddagger \quad$ KoCo19 study group: Emad Alamoudi, Jared Anderson, Valeria Baldassare, Maximilian Baumann, Marc Becker, Marieke Behlen, Jessica Beyerl, Rebecca Böhnlein, Anna Brauer, Vera Britz, Friedrich Caroli, Lorenzo Contento, Alina Czwienzek, Flora Deák, Emma Dech, Laura Dech, Jana Diekmannshemke, Anna Do, Gerhard Dobler, Jürgen Durner, Ute Eberle, Judith Eckstein, Tabea M. Eser, Philine Falk, Volker Fingerle, Stefanie Fischer, Marius Gasser, Sonja Gauder, Otto Geisenberger, Christof Geldmacher, Leonard Gilberg, Kristina Gillig, Philipp Girl, Elias Golschan, Vitus Grauvogl, Celina Halfmann, Tim Haselwarter, Arlett Heiber, Matthias Herrmann, Stefan Hillmann, Christian Hinske, Janna Hoefflin, Tim Hofberger, Michael Höfinger, Larissa Hofmann, Sacha Horn, Kristina Huber, Christian Janke, Ursula Kappl, Charlotte Kiani, Isabel Klugherz, Norah Kreider, Arne Kroidl, Magdalena Lang, Clemens Lang, Silvan Lange, Ekaterina Lapteva, Michael Laxy, Reiner Leidl, Felix Lindner, Alexander Maczka, Alisa Markgraf, Paula Matcau, Rebecca Mayrhofer, Anna-Maria Mekota, Hannah Müller, Katharina Müller, Leonie Pattard, Claire Pleimelding, Stephan Prückner, Konstantin Pusl, Elba Raimúndez, Julius Raschka, Jakob Reich, Raquel Rubio-Acero, Nicole Schäfer, Paul Schandelmaier, Lara Schneider, Sophie Schultz, Mirjam Schunk, 
Lars Schwettmann, Heidi Seibold, Paul Stapor, Jeni Tang, Fabian Theis, Sophie Thiesbrummel, Eva Thumser, Niklas Thur, Julian Ullrich, Julia Waibel, Claudia Wallrauch, Julia Wolff, Pia Wullinger, Tobias Würfel, Patrick Wustrow, Houda Yaqine, Sabine Zange, Eleftheria Zeggini, Thorbjörn Zimmer,

Thomas Zimmermann, Lea Zuche.

\begin{abstract}
Given the large number of mild or asymptomatic SARS-CoV-2 cases, only population-based studies can provide reliable estimates of the magnitude of the pandemic. We therefore aimed to assess the sero-prevalence of SARS-CoV-2 in the Munich general population after the first wave of the pandemic. For this purpose, we drew a representative sample of 2994 private households and invited household members 14 years and older to complete questionnaires and to provide blood samples. SARS-CoV-2 seropositivity was defined as Roche N pan-Ig $\geq 0.4218$. We adjusted the prevalence for the sampling design, sensitivity, and specificity. We investigated risk factors for SARS-CoV-2 seropositivity and geospatial transmission patterns by generalized linear mixed models and permutation tests. Seropositivity for SARS-CoV-2-specific antibodies was $1.82 \%$ (95\% confidence interval (CI) $1.28-2.37 \%$ ) as compared to $0.46 \%$ PCR-positive cases officially registered in Munich. Loss of the sense of smell or taste was associated with seropositivity (odds ratio (OR) 47.4; 95\% CI 7.2-307.0) and infections clustered within households. By this first population-based study on SARS-CoV-2 prevalence in a large German municipality not affected by a superspreading event, we could show that at least one in four cases in private households was reported and known to the health authorities. These results will help authorities to estimate the true burden of disease in the population and to take evidence-based decisions on public health measures.
\end{abstract}

Keywords: COVID-19; SARS-CoV-2; population-based cohort study; seroprevalence; infection fatality ratio; underreporting

\title{
1. Introduction
}

The COVID-19 pandemic has changed life across the globe. The global case number, based on positive PCR results, is ever increasing. In Germany, the first COVID-19 case was diagnosed in Munich on 27 January 2020 [1]. While further spread could be limited to a cluster of 15 co-workers and their families by thorough contact tracing and quarantine, the next cases were detected only 5 weeks later. Following this, the number of SARS$\mathrm{CoV}-2$-infected individuals in Munich increased exponentially from 44 cases diagnosed by 7 March to 3304 cases diagnosed by 3 April 2020 [2]. Overall, Germany had the second highest number of registered COVID-19 cases in Europe in late February 2020, surpassed only by Italy. Accordingly, the mitigation stage of the German National Pandemic Plan was implemented, including an 8 week long "lockdown" beginning on March 16 with restrictions such as school, shop, restaurant and hotel closures in 14 of the 16 German federal states, including Bavaria. With these measures, the first wave of the pandemic was considered under control by early May 2020 and a step-by-step relaxation of public health measures followed. At the same time, public health measures such as the use of facemask was made obligatory in public places in Bavaria and other federal states of Germany. The second wave of the pandemic started in Germany in early October 2020. This time, a "lockdown light" was implemented, during which, e.g., schools and shops were left open, resulting in hospitals reaching their capacity limit in several parts of Germany. In Munich, case numbers rose to 8136 active infections registered by the health authorities on 19 December 2020. Schools and shops were hence again closed in all parts of the country from 16 December 2020 to 8 March 2021. In parallel, vaccination started targeting the highest risk group (people over the age of 80 years, residents of homes for the elderly, healthcare workers at highest risk of infection). Starting from 6 March 2021, self-testing was added to the public health measures aiming at the control of the pandemic in Germany [3]. 
The numbers given above are based on official case reports. However, the number of asymptomatic individuals or patients with mild symptoms not reporting to the health system is largely unknown. This knowledge is crucial to estimate the burden of disease in a population, including true reproduction numbers and attack rates [4]. Up to now, only limited peer-reviewed data from population-based cross-sectional serosurveys on SARS-CoV-2 antibody prevalence at different time points are available. A meta-analysis also evaluating pre-prints of seroprevalence studies [5] reported seroprevalences ranged from below $1 \%$ in Iceland to more than $30 \%$ in Guilan province, Iran [6,7].

In Germany, one population-based study was conducted following a superspreading event in a smaller town, showing an adjusted immunoglobulin $\mathrm{G}(\mathrm{Ig} G)$ seroprevalence close to $20 \%$ [8]. These previous serosurveys used diverse sampling as well as testing methods with varying validity, affecting comparability as well as interpretability of results [3]. Although testing for SARS-CoV-2-specific antibodies seems superior to PCR testing for defining the real dimension of past infections, high sensitivity and especially specificity are crucial due to the low prevalence at the population level [9].

We therefore aimed to identify the complete SARS-CoV-2 seroprevalence in Munich private households including asymptomatic persons and mildly affected patients not reporting to the healthcare system. By doing so, we aimed to provide health authorities with information on the population still at risk for SARS-CoV-2 infections after the first wave of the pandemic.

\section{Methods}

Study design, setting, and population. A detailed description of the study design, setting, and population was previously published [10]; details on sampling design and statistical considerations are given in Online Text S1. In short, we carried out the fieldwork for this baseline of a future cohort study between 5 April and 12 June 2020, in which we selected a random sample of 100 out of 755 Munich constituencies as starting points to represent the Munich population (Figure 1A). Using random route methodology, fieldworkers selected approximately 30 households per constituency, starting from the city's geographic center. They partly crossed the borders of constituencies, resulting in a selection of 2994 households in 368 of the 755 constituencies (Figure 1B). If multi-party houses were selected, we aimed to include 1 household per floor to study transmission dynamics within buildings. The mean number of households recruited per house varied between 1 and 7 across constituencies (Figure 1C). In each selected household, all members aged 14 years and above were invited to participate in the study to assess within-household transmissions (Figure 1D).

Specimen collection and laboratory analyses. During study visits at each household, fieldworkers collected venous blood samples using Ethylenediamine Tetraacetic Acid (EDTA) tubes from each consenting study participant 14 years and older. For ethical reasons, younger children could not provide venous blood samples at this stage. All laboratory methods are described in a previously published preprint [11]. In brief, we determined antibody reactivity using Anti-SARS-CoV-2-ELISA for IgG/IgA (Euroimmun Anti-S1-SARS-CoV-2-ELISA-IgG, hereafter EI-S1-IgG/Euroimmun Anti-S1-SARS-CoV-2ELISA-IgA, hereafter EI-S1-IgA), and the Elecsys Anti-SARS-CoV-2 Roche anti-N pan-Ig (hereafter Ro-N-Ig). We also used the GenScript cPass assay. For serological confirmation, we used a virus micro-neutralization test as described previously [12]. To obtain accurate seroprevalences, we performed validation studies using a panel of 991 truly SARS-CoV-2negative plasma samples from the pre-COVID-19 era and 193 samples from PCR-confirmed COVID-19 patients [11].

While the agreement between EI-S1-IgG and Ro-N-Ig assays was generally high (Online Text S2, Figure S1), the latter gave more valid results. For this assay, an optimized cutoff of 0.4218 (instead of 1.0 ) yielded a sensitivity of $88.60 \%$ and a specificity of $99.72 \%$ [11]. We therefore used the Ro-N-Ig assay with this optimized cut-off to determine seropositivity for SARS-CoV-2 in our analyses. Robustness of the prevalence estimates was tested with 
EI-S1-IgG, EI-S1-IgA, and combinations of different assays as well as different cut-offs and measures of test performance to predict seropositivity (Online Figures S1, S2 and S4, and Table S4).

A

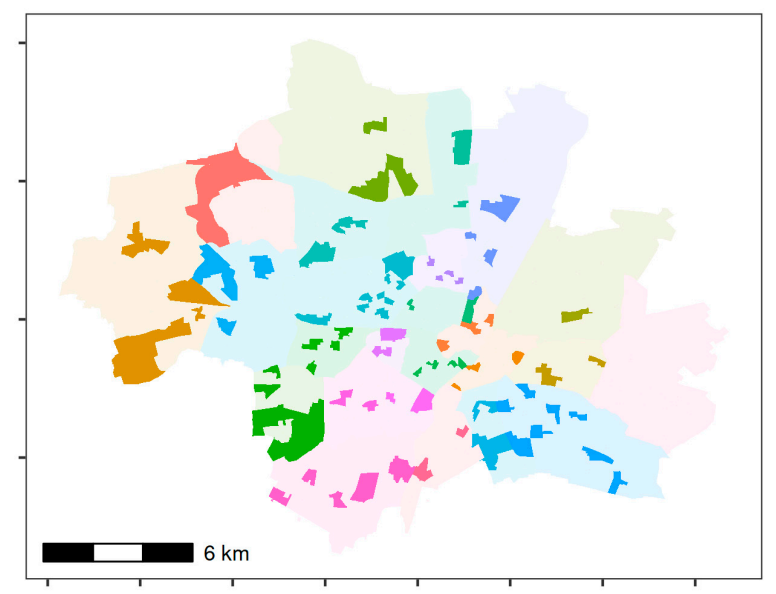

Random selection of constituencies $(n=100)$

\section{C}

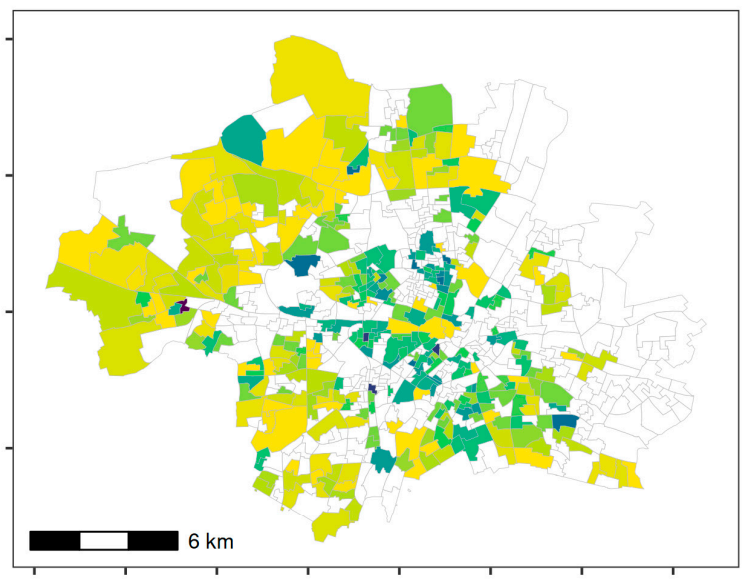

Average number of households per house

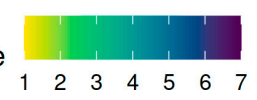

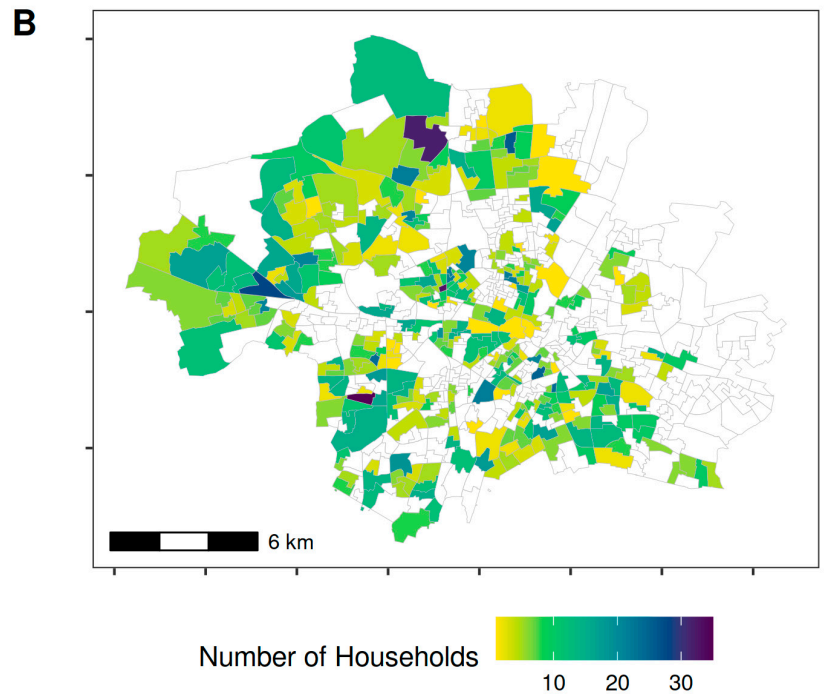

D

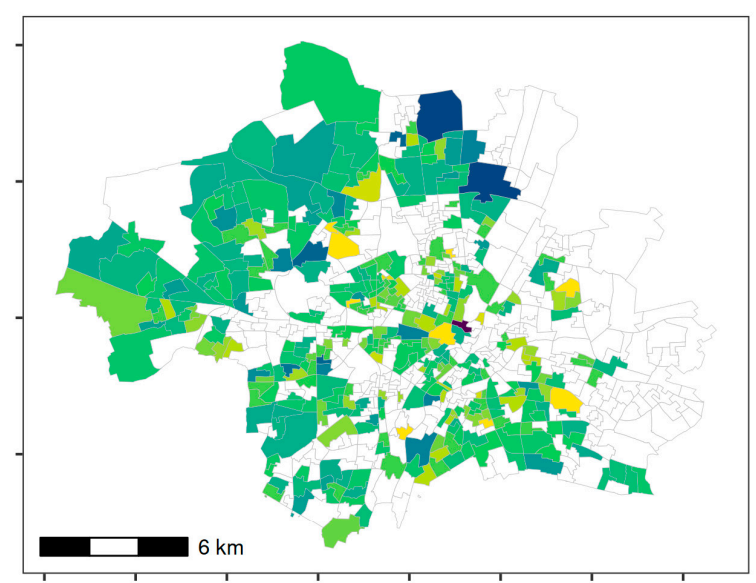

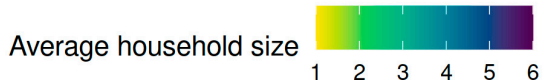

Figure 1. Selection procedure and geospatial distribution of the study population. (A) The municipality of Munich together with its districts (distinguished by different colors). The 100 selected start constituencies for the random walks are marked in the same color as the respective constituency but in a darker shade. (B) All 2994 included households and their respective 368 constituencies. (C) Average number of recruited households per building by constituency. (D) Average number of members per recruited household by constituency.

Household and personal data collection. During household visits, field workers used the mobile data collection tool OpenDataKit (ODK) to capture contact details of household members on Android smartphones. Participants completed a household form and a personal questionnaire online using a newly developed web-based application. Non-responders were reminded by email no later than 2 weeks after the household visit, followed by telephone reminders. Telephone interviews were offered to those who felt unable to complete the questionnaires online.

Statistical analyses. All statistical analyses were performed using the statistical software R (version 4.0.2, R Development Core Team, 2020). 
We calculated absolute and relative frequencies of sociodemographic and household variables and compared them to data of the general Munich population. Online and telephone responses were compared using Fisher's exact test if there were only 2 categories and chi-squared test if there were more than 2 categories.

To assess the seroprevalence, we defined SARS-CoV-2 seropositivity on the basis of the Ro-N-Ig test result, applying the optimized cut-off of 0.4218 as described above. To account for the sampling design, we computed the sampling weights and calibrated them such that the sample structure mirrored the Munich population (regarding age, sex, migration background, presence of children in the households, single-member households). No spatial autocorrelation was assumed as the calculation of Moran' I was not statistically significant. Therefore, in our different analyses, we did not account for the spatial autocorrelation (e.g., at the district level; Online Figure S3). Prevalence estimates were calculated using the calibrated weights, and $95 \%$ confidence intervals were computed on the basis of the variability associated with the sampling design (Online Figure S8). These prevalence estimates were additionally adjusted for sensitivity and specificity of the test as described by Sempos and colleagues (for details on sampling design and sensitivity/specificity adjustments, see Online Text S1) [9]. We calculated the infection fatality ratio (IFR) for the population aged 14 years and older using the seroprevalence estimates for Munich as described above and officially reported numbers of COVID-19 deaths. Fatality counts follow a binomial distribution with small success probability parameter and relatively large number of trials, and thus can be approximately described by a Poisson distribution. This assumption leads to estimates of seroprevalence and corresponding 95\% confidence intervals (CI), both for the entire period of our study as well as for weekly incidences. Official numbers include both subjects living in private households and institutions (e.g., homes for the elderly). To the best of our knowledge, there are no data for Munich on the percentages of SARS-CoV-2 infections and deaths occurring in institutions. For Germany, the Robert Koch Institute (RKI) reported that up to the end of the study period, $13 \%$ of infections and $46 \%$ of deaths occurred in institutions [13]. Given the lack of data for Munich, we estimated the IFR for Munich assuming that the percentage of deaths occurring in members of private households lay in the range between 20 and 100. Likewise, we calculated the factor of underreported infections assuming that between 20 and $100 \%$ of reported infections occurred outside institutions (Online Text S1).

In the risk factor analyses, associations between the personal and household level covariates and seropositivity were evaluated using logistic regression, i.e., generalized linear models (GLM). We adjusted for age and sex and assumed item-nonresponse to be missing at random. The computed odds ratios (ORs) and 95\% CIs were compared to the results of generalized linear mixed models (GLMM), which allowed us to consider the effect of household clustering during the estimation process [14]. For sensitivity analysis, we imputed missing values (of covariates) under the (Bayesian) joint analysis and imputation of incomplete data (JointAI) framework, which allowed us to avoid pooling [15]. The identified important risk factors for seropositivity were included in multiple regression models. Specifically, we compared the frequentist setup of GLM and GLMM as well as a GLMM using simultaneous imputation of multiple missing covariates under the Bayesian framework (Online Text S3, Figures S5 and S6).

To analyze clustering of SARS-CoV-2 infections, we used the similarity of seropositivity levels within spatial clusters of different sizes, i.e., households, buildings, and geospatial clusters of various distances. As a test statistic, we employed the average within-cluster variance. To assess significance, we performed a non-parametric approximate permutation test with $n=10,000$ randomly permuted measurement assignments [16]. To account for household clustering, when analyzing buildings and geospatial clusters, we only permuted households of the same size (Online Text S4).

For official incidence and mortality, as well as for data on the general population, we used data provided by the Statistical Office of the City of Munich. 


\section{Results}

Description of the study samples and population. Of the 6896 households identified, 4903 were eligible and 2994 were included in the analyses. Within these households, fieldworkers invited 6117 persons to participate, of which 5313 agreed and provided blood samples (Figure 2).

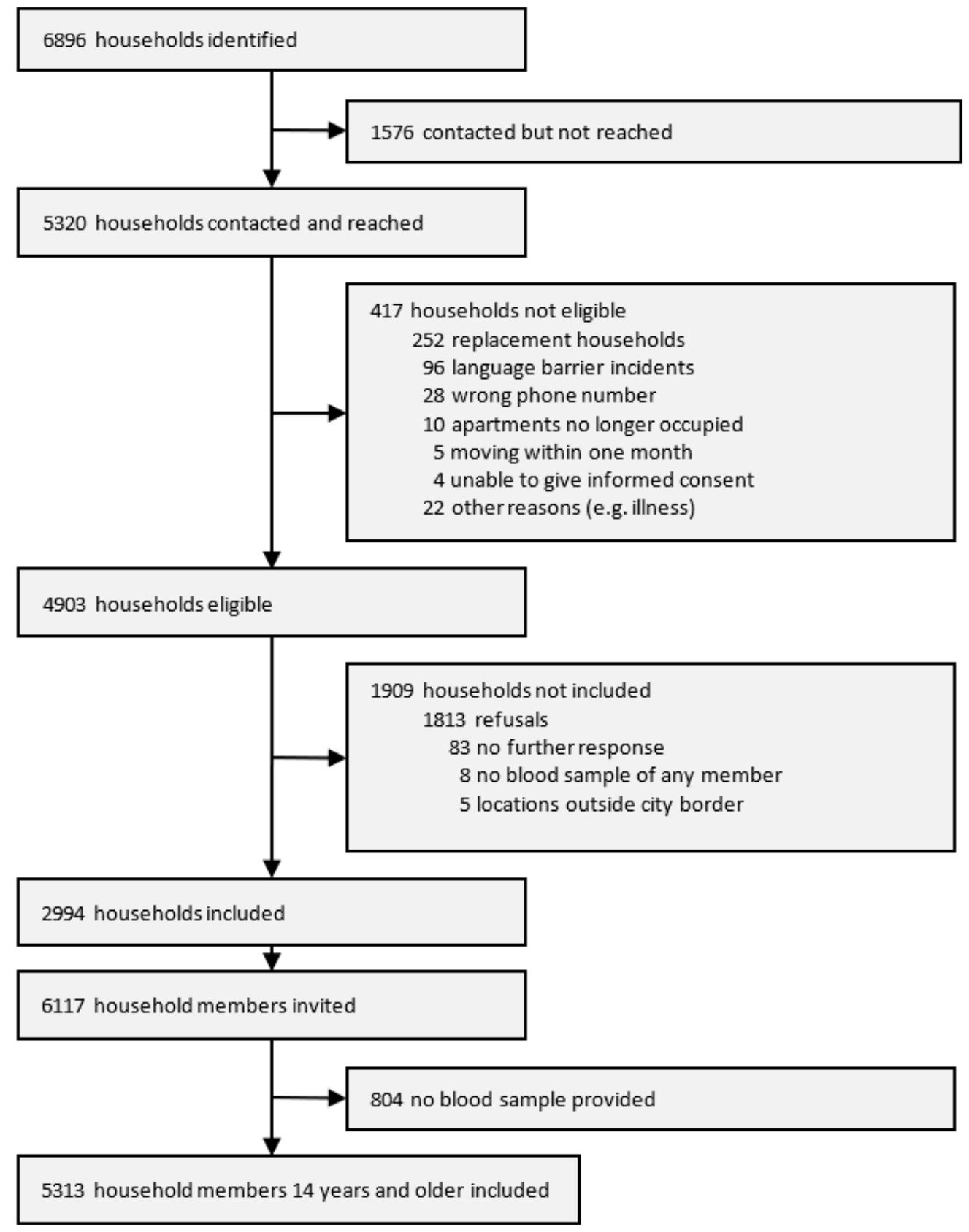

Figure 2. Flow chart on participant selection for the KoCo19 baseline survey.

The study population was comparable to the Munich population with respect to sex ( $52 \%$ vs. $50 \%$ women) (Table 1 ). However, it contained less children and adolescents $(5 \%)$ than the general population (17\%) as children younger than 14 years had been excluded. In addition, persons born outside Germany were underrepresented (18\% in the study population vs. $31 \%$ in the general population). Regarding household characteristics, the sampling design resulted in a preference for larger apartment buildings with $71 \%$ of the 
study population living in apartment houses with five or more apartments compared to $34 \%$ of the Munich population.

Table 1. Individual and household characteristics of the KoCo19 study participants compared to the Munich population.

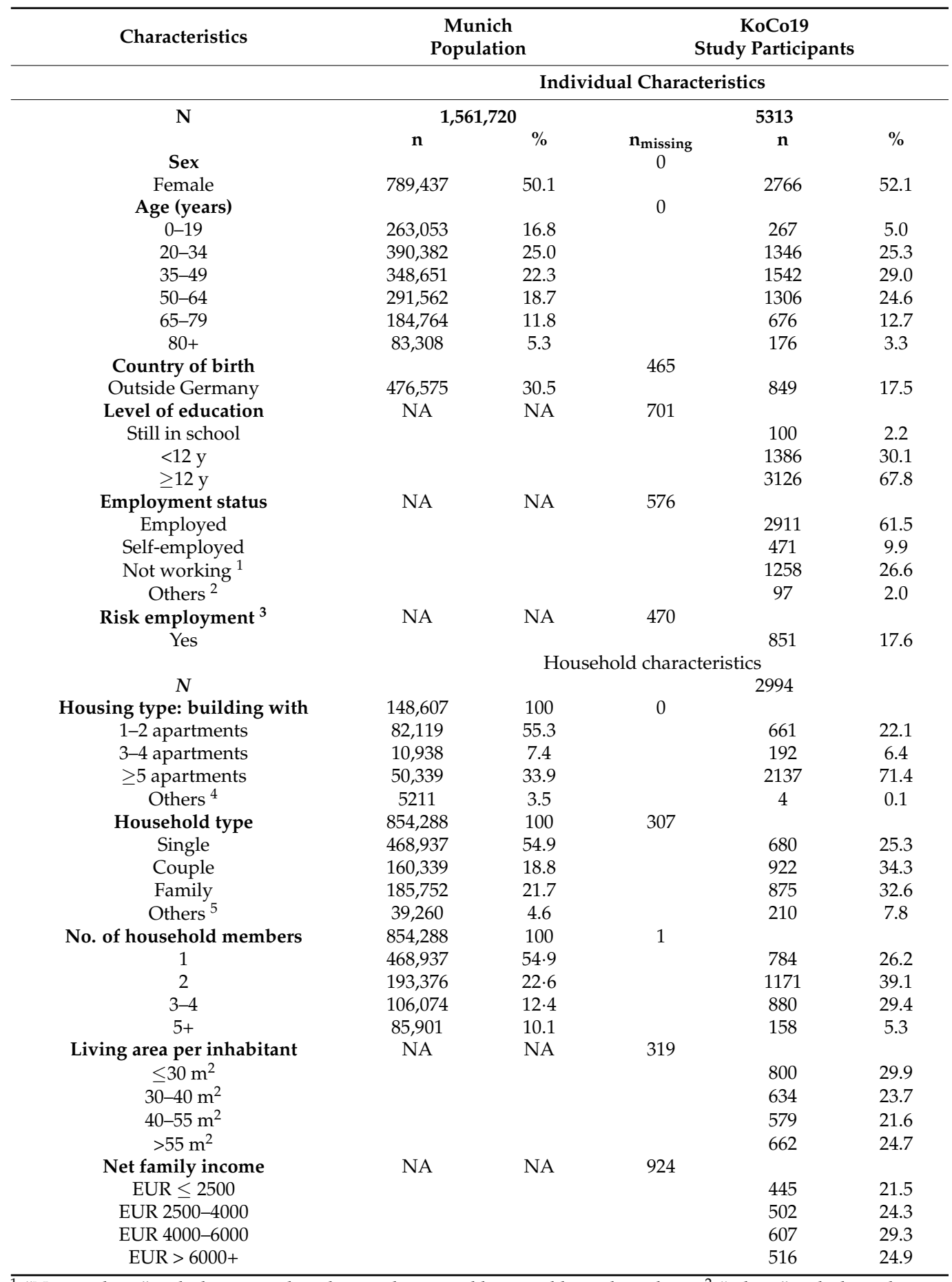

1 "Not working" includes unemployed, retired, parental leave, sabbatical, students; ${ }^{2}$ "others" includes voluntary social year, military service, part-time jobber, reduced working hours; ${ }^{3}$ considered as "risk employment" for COVID-19 infections were employees in the healthcare sector, emergency service, senior homes, airport, public transport, education, sales, social work, and other risk jobs; ${ }^{4}$ other types of housing include tents, caravans, or the like; ${ }^{5}$ other household types include shared apartments by, e.g., students, subleasing, and assisted accommodation. 
Some groups were more likely to participate in telephone interviews than to complete the online questionnaire-women (59\% of telephone interviewees vs. $52 \%$ of online interviewees), participants aged $65+$ years $(66 \%$ vs. $12 \%)$, and subjects with a lower level of education $(72 \%$ vs. $26 \%)$. These marked differences were also reflected in other personal characteristics such as employment status and smoking behavior, as well as in health-related-parameters (Online Table S1).

Prevalence of SARS-CoV-2-specific antibodies and mortality. The highest number of PCR-positive individuals reported by the Statistical Office of the City of Munich was registered in March 2020 before the start of the fieldwork, reaching a maximum in week 14 (Figure 3A,D). The overall number of PCR-positive individuals 14 years and older from the beginning of March until the end of the fieldwork was 6293. This corresponded to a positivity rate of $0.46 \%$. We began the fieldwork in week 15 and ended in week 24 (Figure 3B). Over these weeks, official case numbers went markedly down. In contrast, Ro-N-Ig seroprevalence stayed stable throughout the study period (Figure 3E and Online Figure S7). Using the optimized cut-off, the crude Ro-N-Ig seropositivity for the whole study population was $1.75 \%$ (95\% CI 1.28-2.22\%), with similar results when accounting for the sampling design using calibrated weights (1.89\%; $95 \%$ CI 1.41-2.37\%). Adjusting for test sensitivity and specificity slightly lowered the prevalence estimate (unweighted: $1.67 \%$, CI 1.13-2.20\%; weighted: $1.82 \%$, CI 1.28-2.37\%) (Online Figure S3). The estimated number of cases was about fourfold the official number (Figure 3C). Assuming (on the basis of data for the whole of Germany) that $87 \%$ of infections occurred in private households, we found that 4.5 times (95\% CI 3.2-5.9) more infections than reported occurred (for details, see Online Text S1).

On the basis of official mortality data for the Munich population, we observed an excess mortality in weeks 14 to 19, 2020, compared to the previous 4 years (Figure 3G,H). The resulting overall number of deaths over the whole period was similar to the 216 COVID19 deaths reported by the Statistical Office of Munich (Figure 3I). Assuming the weighted and adjusted Ro-N-Ig prevalence of $1.82 \%$ for the Munich population 14 years and older $(1,369,444$ inhabitants), we estimated that until the end of the field work, 24,990 individuals developed SARS-CoV-2 antibodies (for details on this and the following calculations, see Online Text S1). Up to the end of the fieldwork, the 216 registered COVID-19-related deaths yielded an IFR of $0.86 \%$ (95\% CI: $0.67-1.23 \%$ ) (Figure 3F). Estimating that only $54 \%$ of them occurred in private households, IFR lowered to $0.47 \%$ (95\% CI: $0.36-0.67 \%$ ).

Associations with SARS-CoV-2 seropositivity. Bivariate analyses suggested that RoN-Ig seropositivity depended only weakly on most surveyed factors. Loss of the sense of smell or taste at the time of the study was associated with the outcome; however, the confidence interval was wide (OR 41.3; 95\% CI 6.7-231.0) using a classical GLMM. In addition, respiratory allergies (OR 3.3; 95\% CI 1.1-10.3) were statistically significantly associated with Ro-N-Ig seropositivity. Only weakly related to the outcome were those working in a high-risk job, household type, and living area per inhabitant (Figure 4 and Online Table S2). Besides the loss of the sense of smell or taste, which was considered a symptom of the outcome rather than a risk factor, we included these variables in the final GLMM in which none of the associations were statistically significant. When we applied Bayesian GLMM with imputation of missing values in the sensitivity analyses, we obtained similar results (Figure 5 and Online Table S3).

SARS-CoV-2 transmission within households, buildings, and neighborhoods. Ro$\mathrm{N}$-Ig test outcomes of participants had a significantly lower variance within households than among the entire population (Figure 6 and Online Text S4, Figure S9, Table S5). In contrast, we did not find a statistically lower variance within buildings $(p=0.26)$ nor within neighborhoods applying radii from $50(p=0.16)$ to $4000 \mathrm{~m}(p=0.78)$. Yet, a lower-thanexpected mean variance was seen up to a distance of $200 \mathrm{~m}$. 

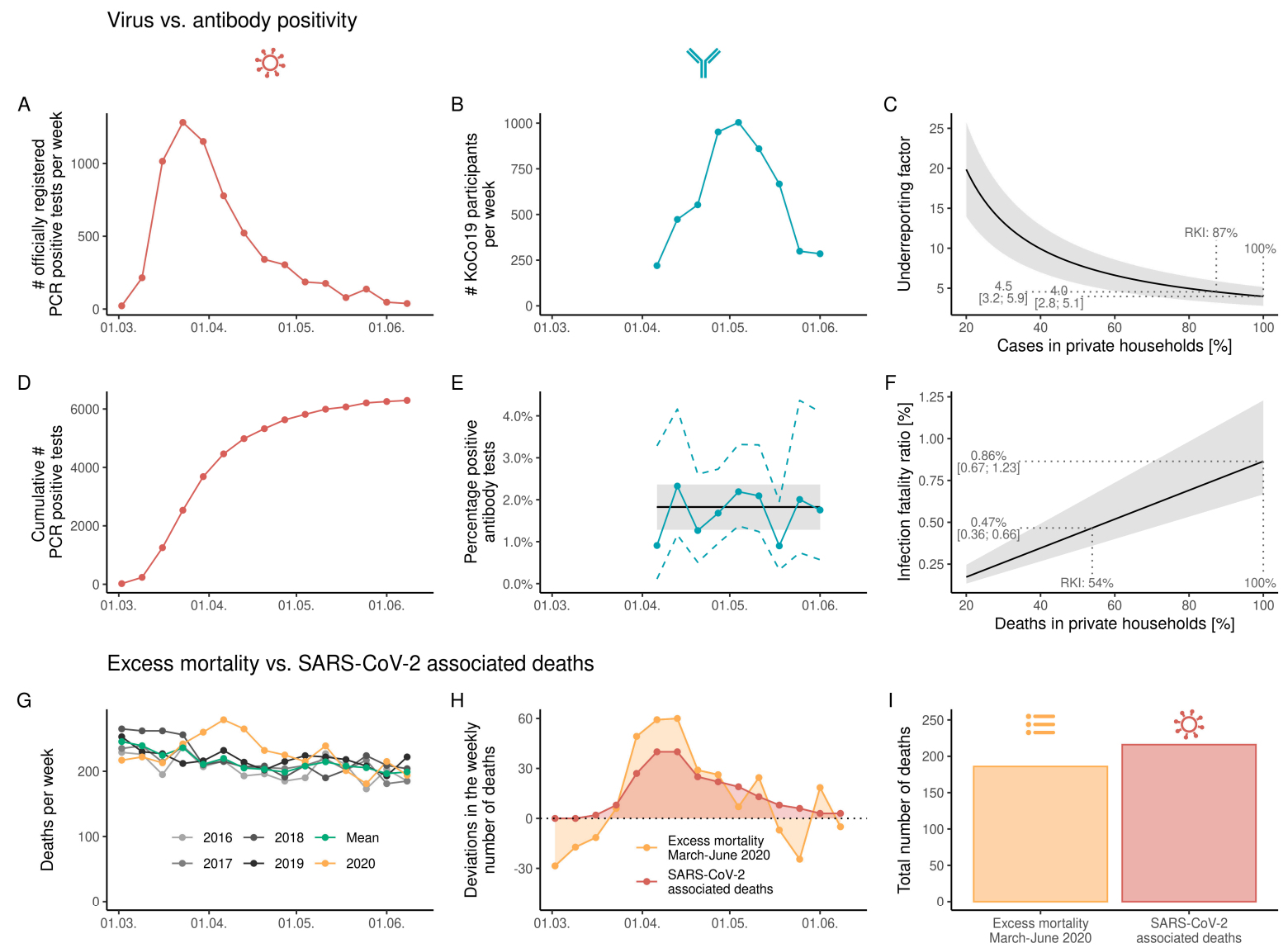

Figure 3. Dynamics of the COVID-19 pandemic and of the KoCo19 study in Munich since the beginning of the pandemic to the end of the KoCo19 study period. (A) Official weekly absolute number of newly diagnosed COVID-19 cases based on positive PCR tests. (B) Weekly number of participants recruited to the KoCo19 study. (C) Estimated underreporting factor depending on the percentage of reported cases in private households with respect to all reported cases in Munich. (D) Cumulative weekly number of officially registered COVID-19 infections in Munich. (E) Numbers of Elecsys Anti-SARSCoV-2 Roche anti-N pan-Ig (Ro-N-Ig) seropositive samples per week (blue) divided by the number of blood draws in the respective time frame. $95 \%$ CIs (blue dashed lines) are based on an approximate Poisson assumption. Black line and shaded area indicate the weighted and adjusted prevalence estimate with $95 \%$ CI. Due to low recruitment numbers in the last week, in (D,E), the data from the last week were integrated with the pre-last week. (F) Estimated infection fatality ratio depending on the percentage of reported COVID-19-related deaths in private households with respect to all reported COVID-19-related deaths in Munich. (G) Weekly number of deaths in Munich for 2016-2020 in terms of official numbers. (H) Weekly excess mortality in 2020 compared to 2016-2019 in terms of official death counts and official SARS-CoV-2-related deaths. (I) Comparison of total number of deaths in terms of excess mortality and registered SARS-CoV-2-related deaths. 


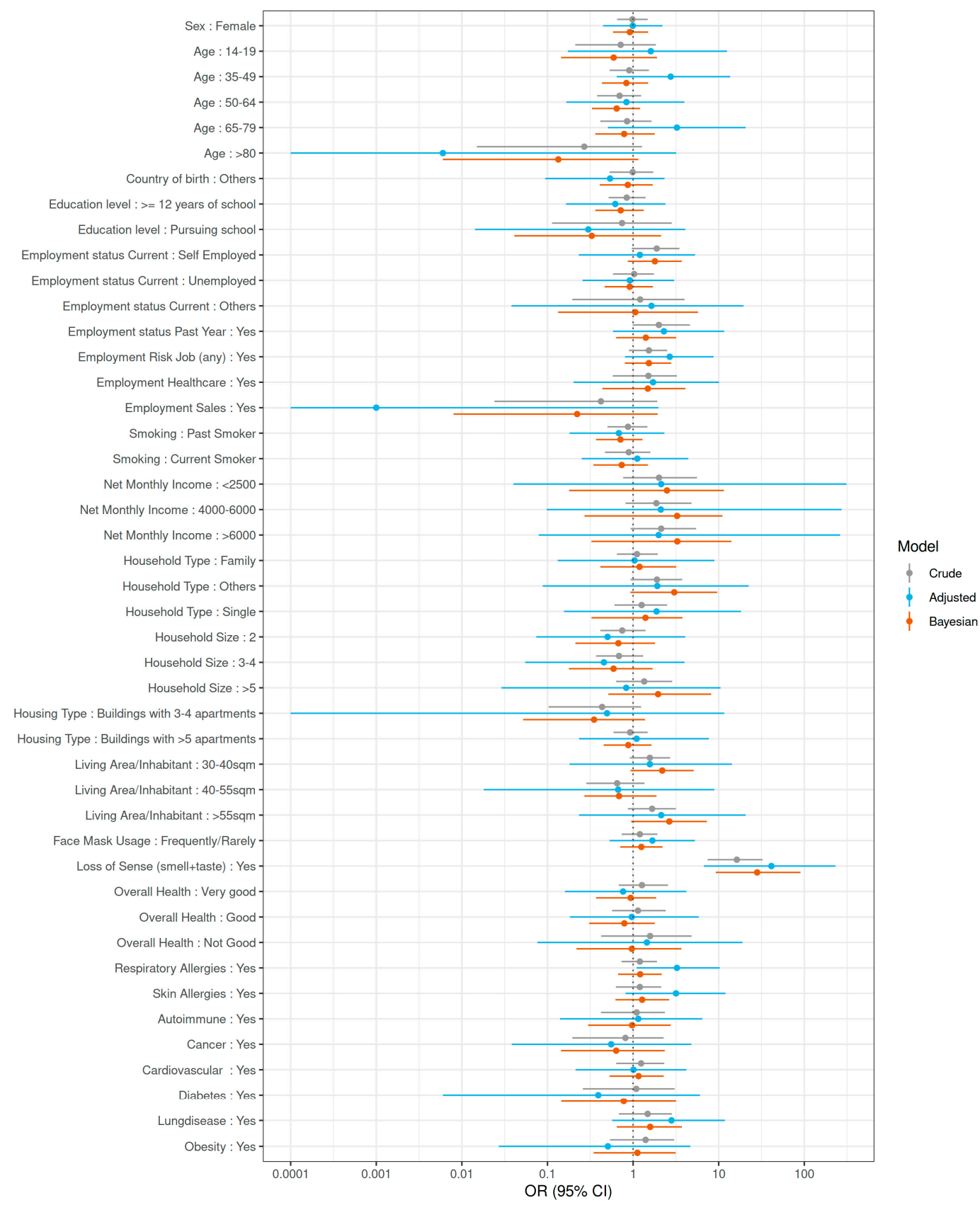

Figure 4. Risk factor analysis for SARS $\neg \mathrm{CoV}-2$ seropositivity. Risk factor analysis for SARS $\neg$ CoV-2 seropositivity in the KoCo19 study population comparing crude, adjusted for clustering, and Bayesian (after imputation and adjusted for clustering) estimates. All odds ratios (ORs) and 95\% CIs were adjusted for age (continuous scale) and sex. OR: odds ratio; 95\% CI: 95\% confidence interval (frequentist GLMM)/95\% credible interval (Bayesian analyses). 


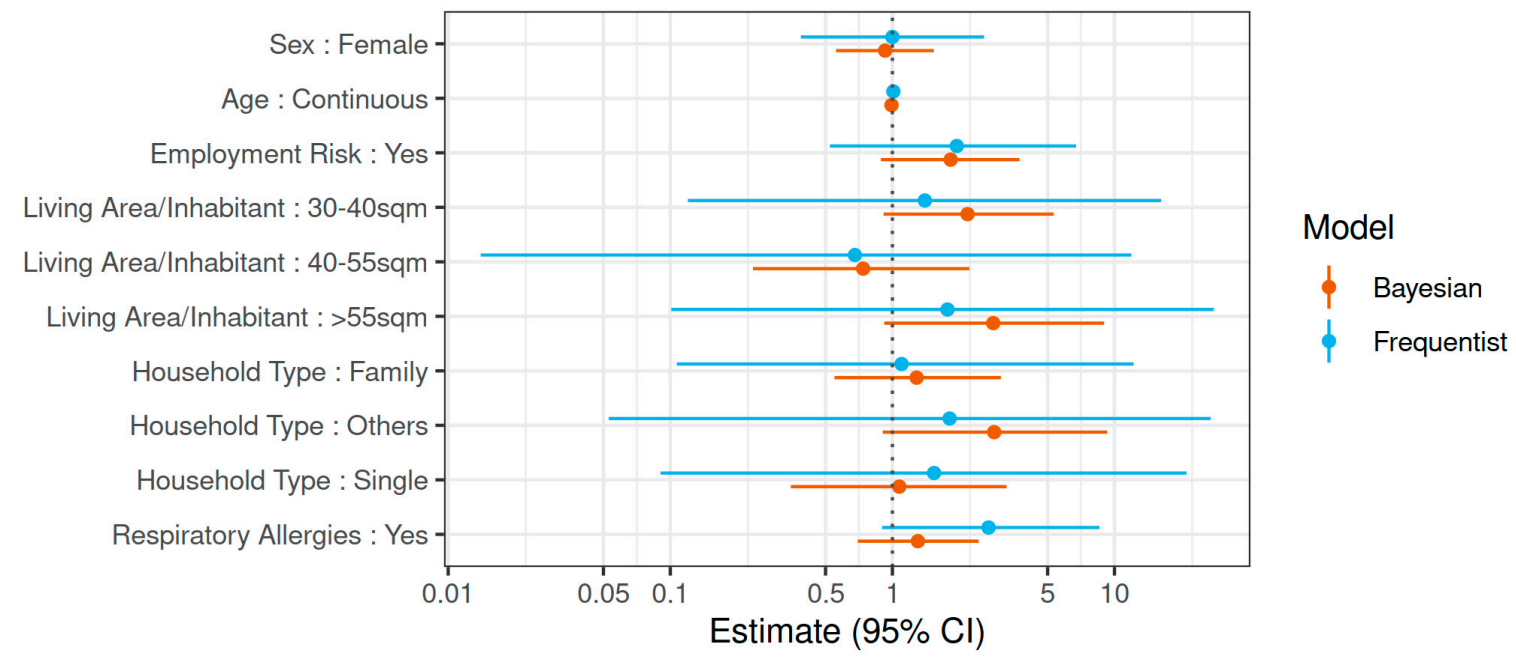

Figure 5. Multivariate risk factor analysis for SARS $\neg \mathrm{CoV}-2$ seropositivity. Multivariate risk factor analysis for SARS-CoV-2 seropositivity mutually adjusted for all variables in the figure. OR: odds ratio; 95\% CI: 95\% credible interval (Bayesian analyses) $/ 95 \%$ confidence interval (frequentist GLMM).

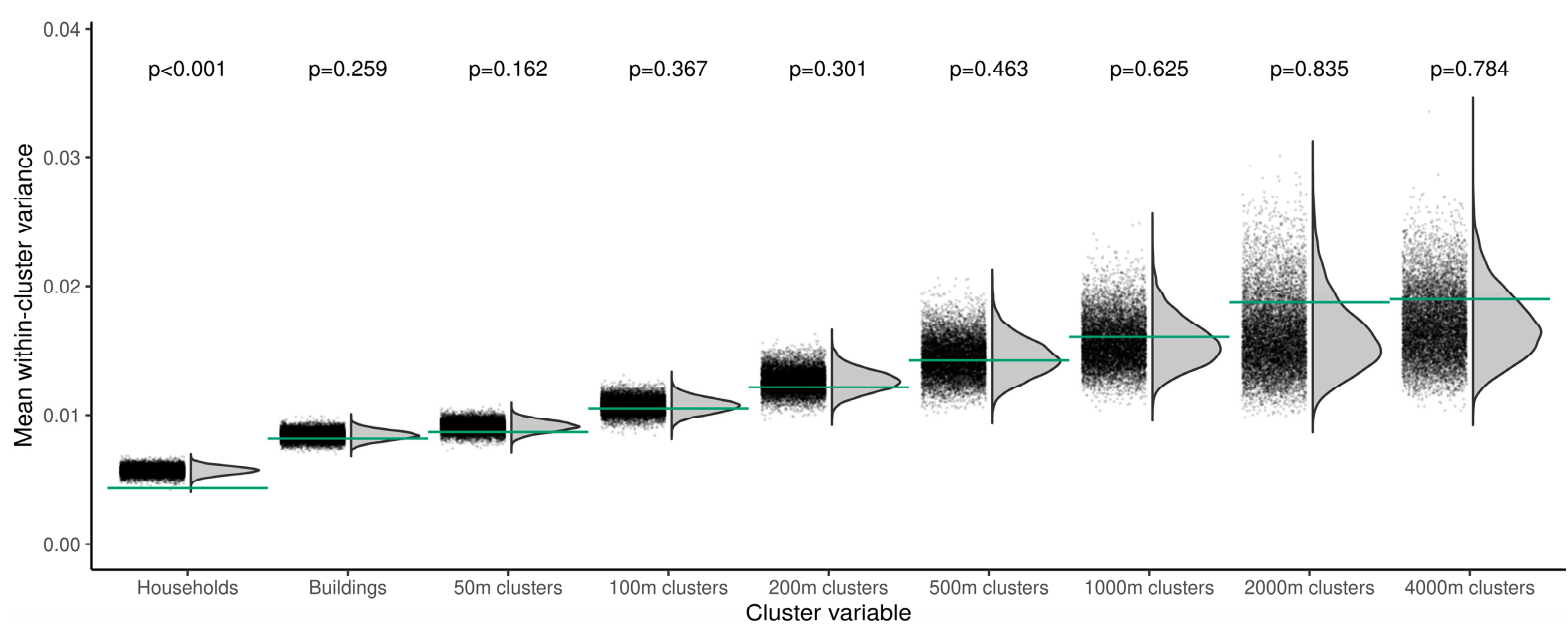

Figure 6. Proximity clustering of Ro-N-Ig test outcomes. We subdivide the participants into disjoint clusters according to various cluster definitions: households, buildings, and spatial clusters of various diameters ( $x$-axis). For each cluster, we calculated the within-cluster variance of observed Ro-N-Ig test outcomes of all participants in the cluster. Their means over all clusters are marked by green horizontal lines for each cluster size. We then performed 10,000 random permutations of measurements assignments. The black dots show the respective mean within-cluster variances, along with density estimates as grey curves. For buildings and spatial clusters, measurements of a household were only permuted with measurements of a household of the same size. $p$-values indicate the one-sided probability of a random value being smaller than or equal to the observed one.

\section{Discussion}

We present an estimate for the SARS-CoV-2 seroprevalence in the Munich general population 14 years and older, which was still low towards the end of the first pandemic wave $(1.82 \%)$. However, our results indicate that the seroprevalence was substantially higher than official numbers in terms of registered PCR-positive cases. We could only identify weak risk factors for SARS-CoV-2 seropositivity. Finally, our data confirmed household clustering of infection [17]. 
As study participants were enrolled at a time when the newly released serological assays were not fully validated, we carefully evaluated three primary serological assays [11] As similarly reported by Gudbjartsson et al., the specificity and sensitivity of Roche anti-N pan-Ig was superior to Euroimmun [6]. By using the cut-off index raw values, we were able to optimize the cut-off of the assay.

Our prevalence estimates are in line with findings from Gudbjartsson et al. who estimated the SARS-CoV-2 antibody seropositivity for the general population of Iceland at around $1 \%$, thus being slightly lower than in our population [6]. Other studies estimating seroprevalence for European general populations reported results between $2 \%$ in Luxembourg [18]; 4\% in Spain [19]; and 11\% in Geneva, Switzerland [20]. The proportion of officially registered cases vs. the number of serologically positive cases also varied significantly between the studies. In our study, about one in four to five seropositive cases were officially registered, although one needs to consider that we did not have data on the seroprevalence among children younger than 14 years. For these, only one out of six infections might have been officially registered, as recently shown by Hippich et al. [21] for children living in Bavaria, Germany. In addition, our study population did not include institutions in which residents might be more frequently tested than in other settings. Therefore, our estimate might even be conservative for the Munich overall population. In other population-based European studies, $10-56 \%$ of infections were detected by the healthcare system $[6,19,20]$. We believe that the proportion of registered vs. seropositive but not registered individuals could be used to measure the efficiency of a public health testing system.

While only a small proportion of the general population in Germany live in institutions, official data of the Robert Koch Institute (RKI) report that during the study period, $13 \%$ of reported SARS-CoV-2 infections and $46 \%$ of COVID-19-related deaths occurred in institutionalized persons [13]. Therefore, we report a range of IFRs depending on the percentage of deaths occurring in private households. Translating the RKI numbers to Munich, the IFR resulting from our study was $0.47 \%$ (95\% CI 0.36-0.67\%). IFR calculations are difficult to compare for the reasons mentioned above and, e.g., due to different approaches or unequal case ascertainment. The IFR resulting from our study is in line with recent data from Geneva, Switzerland $(0.64 ; 95 \%$ CI $0.38-0.98 \%)$, but slightly lower than data reported for Spain $(0.83 ; 95 \%$ 0.78-0.89) [22,23].

Among the risk factors identified, albeit not statistically significant, the increased risk of working in a job with a high potential of contact to COVID-19 cases appears plausible, and the result is in line with other studies [6,19,24]. Among the COVID-19 cases reported globally to the World Health Organization, $14 \%$ belong to the group of healthcare workers, whereas this group represents less than $3 \%$ of the general population in most countries [25]. Special preventive efforts should therefore be always targeting the group of workers occupationally exposed to SARS-CoV-2. We saw slightly increased odds of seropositivity among participants with respiratory co-morbidity, especially patients with allergies. Angiotensin-converting enzyme 2 (ACE2) receptors are thought to be main cell entries for SARS-CoV-2. Differences in expression levels of ACE2 in patients with allergic asthma might be one reason for the increased risk of SARS-CoV-2 seropositivity in our study population [26]. However, our data on respiratory allergies were self-reported, and therefore specificity might be low.

Participants with SARS-CoV-2 antibody responses more frequently reported the loss of the sense of smell or taste, even though the questionnaire was answered up to several weeks after the blood samples, which is consistent with other studies [27]. The underlying pathological mechanism might again be explained by the high concentration of ACE2 in olfactory cells [27]. 
Household clustering was already described for SARS-CoV-2 and is well known from other respiratory infections $[17,24,28,29]$. Apart from transmission from one person to the other within one household, similar behavior and contacts outside their own household might explain this. Our results weakly suggest clustering within the same house and eventually the close neighborhood. However, these associations were not statistically significant and need to be confirmed. With respect to within-household transmission, our seroprevalence observations are limited by the fact that we did not take any blood samples from children below 14 years. Since the number of children in a household is presumably positively correlated with household size, this biases our data, and within-household transmission may even be more pronounced than reported here.

The major strengths of this study are its population-based approach, weighting of results for the general Munich population, the high number of participants, the thorough validation of the assays used, and (where available) the use of validated questionnaire items. The overall response to the study was high compared to other population-based epidemiologic studies in Germany [30]. While most participants completed the questionnaire online, we also provided the alternative of telephone interviews, increasing participation and, thus, making our study population more representative for the target population. Our study started towards the end of the first pandemic wave in Munich, as illustrated by the incidence of SARS-CoV-2 registered cases and mortality. This resulted in a relatively stable antibody prevalence over the course of the fieldwork.

As mentioned, a relevant limitation of our study is the exclusion of children and residents not living in private households. While in general, people with migration background are less likely to participate in population-based studies, the lack of translated questionnaires further limited the number of migrants participating in our study [10]. To increase response, blood samples were collected at participants' homes and not at a centralized testing facility. However, we could not interview participants during home visits. This led to lower questionnaire response and a time interval of up to six weeks between blood sample collection and completion of the questionnaire. Item non-response in online completed questionnaires was higher compared to personal interviews. As in other studies, questions on income ( $21 \%$ missing responses) as well as weight and height ( $73 \%$ missing responses) were especially prone to non-response. They were thus not included in the final models. Despite the high number of participants, the power for our risk factor analyses was relatively low due to the low seroprevalence, resulting in large confidence intervals and potentially negative findings. However, one may assume that any strong risk factors would have been identified if included.

Although commercial and scalable testing methods for SARS-CoV-2 seem robust, cut-offs have to be adapted to specific populations to improve their validity. However, the relevance of antibody-based testing to assess previous infection remains partly unclear. To learn more about the meaning of detected SARS-CoV-2-specific antibodies, correlates of protection, and testing methodologies, this as well as other longitudinal cohort studies need to be continued.

\section{Conclusions}

Even when considering all inherent uncertainties, our results indicate that only a very small proportion of the Munich population encountered SARS-CoV-2 during spring 2020, most citizens stay vulnerable to infection, and the associated risk of death among those infected was high. The careful evaluation of the serum samples contributed to the validity of our results. Methodologically, it provides insights into how a sound populationbased epidemiologic study can be conducted early on in a pandemic. Finally, it is the only population-based study outside a hotspot thus far published in Germany, a country in which early public health measures possibly prevented the healthcare system from collapsing. We therefore consider the data relevant and useful to consider for healthcare providers, also for future pandemics. These results will help authorities to estimate the 
true burden of disease in the population and to make evidence-based decisions on public health measures.

Supplementary Materials: The following are available online at https:/ / www.mdpi.com/article/ 10.3390/ijerph18073572/s1, Text S1: Sampling design and corrections of seroprevalence, Text S2: Details on cut-offs and validation, Text S3: Handling of missing data and risk factor analysis, Text S4: Influence of household size and household clustering, Figure S1: Validation of classifier based on Ro-N-Ig results, Figure S2: Comparison of primary test results (Ro-N-Ig vs EI-S1-IgG) with other tests, Figure S3: Number of participants per city district (A), unweighted (upper figure) and weighted (lower figure) prevalence (\%) of Ro-N-Ig seropositive samples per city district (B) with lower (C) and upper (D) 95\% Confidence Intervals (CI), Figure S4: SARS-CoV-2 seroprevalence obtained by different classifiers for different specificities and sensitivities, Figure S5: Missing patterns observed in our complete analysis data, Figure S6: Missing patterns observed in our multiple regression analysis data with important covariates only, Figure S7: Roche N pan-Ig seroprevalence in the KoCo19 study population unweighted (top) and weighted (bottom), Figure S8: Sampling probabilities for Munich constituencies, Figure S9: Fraction of individuals with positive Ro-N-Ig result for different household sizes, Table S1: Individual characteristics of the KoCo19-study participants comparing telephone interviewees to those answering the online questionnaire, Table S2: Prevalence of Roche N pan-Ig seropositivity and risk factor analysis adjusted for age and sex using three different methods by individual, household and health characteristics of the KoCo19 study population, Table S3: Multivariate risk factor analysis for SARS-CoV-2 seropositivity mutually adjusted for all variables in the table, Table S4: Classifier's specificity and sensitivity depending on the manufacturer's or the optimised cut-offs, Table S5: Overview on the household size distribution, the number of tested individuals per household and the number of positive tests.

Author Contributions: M.H. is the principal investigator of this study and obtained most necessary funds. M.H., K.R. and E.S. conceived the study with input from M.N.D., F.F., J.M.G.N., I.K., L.O., M.P. (Michel Pletschette), M.P. (Michael Pritsch), F.R., V.T. and A.W. Sample collection was led by J.M.G.N., I.K., D.M., L.O., M.P. (Michael Pritsch) and V.T. with assistance from I.B., J.B., J.F., G.F., K.P., C.R. and S.W. The laboratory set-up and sample processing were led by A.W., R.W. was responsible for virus micro-neutralization tests. M.N.D. coordinated the development of the web-based study application with input by E.S. M.N.D., F.F., K.R., F.R., E.S. and S.W. coordinated data acquisition and data management. Data was cleaned and prepared by N.C., M.N.D., F.F. and S.W. Statistical analyses and data visualization were performed by A.B., N.C., T.F., M.G., R.L.G., P.P. and Y.S. C.F. and J.H. led the statistical analyses. C.F., J.H., K.R., F.R., E.S. and S.W. conceptualized the result presentation with input from M.H., I.K., L.O., M.P. (Michael Pritsch) and A.W. The manuscript was primarily written by M.P. (Michael Pritsch) and K.R., with significant contributions from A.B., N.C., T.F., C.F., M.G., R.L.G., J.H., M.H., I.K., L.O., P.P., E.S., Y.S. and A.W. All authors have read and agreed to the published version of the manuscript.

Funding: This study was funded by the Bavarian State Ministry of Science and the Arts, the University Hospital of Ludwig-Maximilians-University Munich, the Helmholtz Centre Munich, the University of Bonn, the University of Bielefeld, and the German Ministry for Education and Research (MoKoCo19, reference number 01KI20271). Euroimmun, Mikrogen, Roche, and Viramed provided kits and machines for analyses at discounted rates. The funders had no role in study design, data collection, data analyses, data interpretation, writing, or submission of this manuscript.

Institutional Review Board Statement: The study was conducted in accordance with good clinical (GCP) and epidemiological practice (GEP) standards as well as the Declaration of Helsinki in its most recent form (as amended by the 64th WMA General Assembly, Fortaleza, Brazil, in October 2013). The study protocol was approved by the Institutional Review Board of the Medical Faculty at Ludwig Maximilian University Munich, Germany (opinion dated 31 March 2020; number 20-275), prior to study initiation.

Informed Consent Statement: Informed consent was obtained from all study participants prior to study inclusion.

Data Availability Statement: Our data are accessible to researchers upon reasonable request to the corresponding author taking data protection laws and privacy of study participants into account. 
Acknowledgments: We gratefully thank all study participants for their trust, time, data, and specimens. We are grateful for the financial support of the Bavarian State Ministry of Science and the Arts, the University Hospital of LMU Munich, the Helmholtz Centre Munich, the University of Bonn, and the University of Bielefeld. J.H. acknowledges financial support by the German Ministry for Education and Research via the project MoKoCo19 (reference number: 01KI20271). M.G. acknowledges support from the Joachim Herz Foundation through the Add-on Fellowship for Interdisciplinary Science. This study would also not have been possible without the staff of the Division of Infectious Diseases and Tropical Medicine at the University Hospital of LMU Munich, Helmholtz Centre Munich, and Bundeswehr Institute of Microbiology, as well as all medical students involved. We thank Judith Eckstein for outstanding support regarding public relations. We thank the teams from the press offices of LMU, University Hospital of LMU Munich, and of Helmholtz Centre Munich. We thank the KoCo19 advisory board members Stefan Endres, Stephanie Jacobs, Bernhard Liebl, Michael Mihatsch, Matthias Tschöp, Manfred Wildner, and Andreas Zapf. We thank Accenture for the development of the KoCo19 web-based survey application. We are grateful to the Statistical Office of the City of Munich, Germany, for providing statistical data on the Munich general population. We thank Helmut Küchenhoff for a critical review of an earlier version of the manuscript and Jared Anderson for English language corrections. We also thank Guillaume Chauvet for advice on the sampling design and variance estimation. We are grateful to the Munich police for their support in the fieldwork. The Munich Surgical Imaging GmbH, Cisco Systems, and the graphic/photo/IT infrastructure departments at the University Hospital of LMU Munich provided support during video production and online events. For fieldwork, BMW Group as part of their campaign "BMW hilft Helfenden" provided free cars. Mercedes-Benz Munich provided support with Mercedes-Benz Rent in the project infrastructure.

Conflicts of Interest: F.F., T.F., D.M., L.O. and V.T. report grants from the Bavarian State Ministry of Science and the Arts during the conduct of the study. T.F. reports grants from the University Hospital of LMU Munich, Helmholtz Center Munich, University of Bonn, University of Bielefeld, and German Ministry for Education and Research during the conduct of the study. J.H. reports grants from the German Federal Ministry of Education and Research during the conduct of the study. M.H. and A.W. report personal fees and non-financial support, L.O. and M.P. report non-financial support from Roche Diagnostics. M.H., L.O., M.P. and A.W. report non-financial support from Euroimmun, Viramed, and Mikrogen. M.H., M.P. and A.W. report grants, non-financial support, and other from German Center for Infection Research (DZIF). F.F., M.H., L.O., M.P., V.T. and A.W. report grants and non-financial support from the Government of Bavaria. M.H., L.O., M.P. and A.W. report non-financial support from BMW, Mercedes Benz, Munich Police, and Accenture. M.H. and A.W. report personal fees and non-financial support from Box Betrobox during the conduct of the study. L.O. and M.P. report non-financial support from Box Betrobox. M.H. and A.W. have a patent Sample System for Sputum Diagnostics of SARS-CoV-2 pending. D.M. reports to be a sub-investigator on a phase I SARS-CoV-2 vaccine trial and on a phase I rabies vaccine trial, both sponsored by CureVac AG. M.P. and A.W. report non-financial support from Becker MVZ. V.T. reports support from CureVac AG outside the submitted work. A.W. reports personal fees and other from Haeraeus Sensors. A.W. reports non-financial support from Bruker Daltronics outside the submitted work. A.W. is involved in other different patents and companies not in relation with the serology of SARS-CoV-2. All other authors report nothing to disclose.Code Availability: To facilitate reproducibility and reuse, we made the code used to perform the analyses and generate the figures available on GitHub (https:/ / github.com/koco19/lab_epi (accessed on 28 March 2021)), and it has been uploaded to ZENODO (http:/ / doi.org/10.5281/zenodo.4300922 (accessed on 28 March 2021)) for long-term storage.

\section{References}

1. Rothe, C.; Schunk, M.; Sothmann, P.; Bretzel, G.; Froeschl, G.; Wallrauch, C.; Zimmer, T.; Thiel, V.; Janke, C.; Guggemos, W.; et al. Transmission of 2019-nCoV Infection from an Asymptomatic Contact in Germany. N. Engl. J. Med. 2020, 382, 970-971. [CrossRef]

2. Coronavirus in München: Die Aktuelle Entwicklung [Internet]. 2020. Available online: https://www.muenchen.de/aktuell/2020 -03/coronavirus-muenchen-infektion-aktueller-stand.html (accessed on 9 October 2020).

3. Bundesministerium für Gesundheit: Coronavirus SARS-CoV-2: Chronik der Bisherigen Maßnahmen [Internet]. 2021. Available online: https: / / www.bundesgesundheitsministerium.de/coronavirus/chronik-coronavirus.html (accessed on 17 March 2021).

4. Pearce, N.; Vandenbroucke, J.P.; VanderWeele, T.J.; Greenland, S. Accurate Statistics on COVID-19 Are Essential for Policy Guidance and Decisions. Am. J. Public Health 2020, 110, 949-951. [CrossRef] [PubMed] 
5. Meyerowitz-Katz, G.; Merone, L. A systematic review and meta-analysis of published research data on COVID-19 infection fatality rates. Int. J. Infect. Dis. 2020, 101, 138-148. [CrossRef]

6. Gudbjartsson, D.F.; Norddahl, G.L.; Melsted, P.; Gunnarsdottir, K.; Holm, H.; Eythorsson, E.; Arnthorsson, A.O.; Helgason, D.; Bjarnadottir, K.; Ingvarsson, R.F.; et al. Humoral Immune Response to SARS-CoV-2 in Iceland. N. Engl. J. Med. 2020, 383, 1724-1734. [CrossRef]

7. Shakiba, M.; Hashemi Nazari, S.S.; Mehrabian, F.; Rezvani, S.M.; Ghasempour, Z.; Heidarzadeh, A. Seroprevalence of COVID19 Virus Infection in Guilan Province, Iran [Internet]. Infectious Diseases (except HIV/AIDS). 2020. Available online: http: / / medrxiv.org/lookup/doi/10.1101/2020.04.26.20079244 (accessed on 5 August 2020).

8. Streeck, H.; Schulte, B.; Kümmerer, B.M.; Richter, E.; Höller, T.; Fuhrmann, C.; Arnthorsson, A.O.; Helgason, D.; Bjarnadottir, K.; Ingvarsson, R.F.; et al. Infection fatality rate of SARS-CoV2 in a su-per-spreading event in Germany. Nat. Commun. 2020, 11, 1-12. [CrossRef]

9. Sempos, C.T.; Tian, L. Adjusting Coronavirus Prevalence Estimates for Laboratory Test Kit Error. Am. J. Epidemiol. 2021, 190, 109-115. [CrossRef]

10. The KoCo19 Collaboration Group; Radon, K.; Saathoff, E.; Pritsch, M.; Noller, J.M.G.; Kroidl, I.; Olbrich, L.; Thiel, V.; Diefenbach, M.; Riess, F.; et al. Protocol of a population-based prospective COVID-19 cohort study Munich, Germany (KoCo19). BMC Public Health 2020, 20, 1-9.

11. Olbrich, L.; Castelletti, N.; Schälte, Y.; Garí, M.; Pütz, P.; Bakuli, A.; Pritsch, M.; Kroidl, I.; Saathoff, E.; Noller, J.M.G.; et al. A Serology Strategy for Epidemiological Studies Based on the Comparison of the Performance of Seven Different Test Systems-The Representative COVID-19 Cohort Munich. medRxiv 2021. [CrossRef]

12. Haselmann, V.; Özçürümez, M.K.; Klawonn, F.; Ast, V.; Gerhards, C.; Eichner, R.L.; Costina, V.; Dobler, G.; Geilenkeuser, W.-J.; Wölfel, R.; et al. Results of the first pilot external quality assessment (EQA) scheme for anti-SARS-CoV2-antibody testing. Clin. Chem. Lab. Med. 2020, 58, 2121-2130. [CrossRef]

13. Robert Koch Institut. Täglicher Lagebericht (13.06.2020) des RKI zur Coronavirus-Krankheit-2019 (COVID-19)—Aktualisierter Stand für Deutschland [Internet]. 2020 [cited 2020 Dec 2]. Available online: https://www.rki.de/DE/Content/InfAZ/N/ Neuartiges_Coronavirus/Situationsberichte/2020-06-13-de.pdf;jsessionid=2A2033699409CB8BA030EB0F22EA0BD4.internet0 62?_blob=publicationFile (accessed on 2 December 2020).

14. Molenberghs, G.; Verbeke, G. Models for Discrete Longitudinal Data; Springer: Berlin/Heidelberg, Germany, 2005 ; p. 683.

15. Erler, N.S.; Rizopoulos, D.; Lesaffre, E.M.E.H. JointAI: Joint Analysis and Imputation of Incomplete Data in R. arXiv 2020, arXiv:190710867.

16. Nichols, T.E.; Holmes, A.P. Nonparametric permutation tests for functional neuroimaging: A primer with examples. Hum. Brain Mapp. 2002, 15, 1-25. [CrossRef]

17. Lee, E.C.; Wada, N.I.; Grabowski, M.K.; Gurley, E.S.; Lessler, J. The engines of SARS-CoV-2 spread. Science 2020, 370 , 406-407. [CrossRef]

18. Snoeck, C.J.; Vaillant, M.; Abdelrahman, T.; Satagopam, V.P.; Turner, J.D.; Beaumont, K.; Gomes, C.P.C.; Fritz, J.V.; Schröder, V.E.; Kaysen, A.; et al. Prevalence of SARS-CoV-2 Infection in the Luxembourgish Population: The CON-VINCE Study. [Internet]. Infectious Diseases (except HIV/AIDS). 2020. Available online: http:/ / medrxiv.org/lookup/doi/10.1101/2020.05.11.20092916 (accessed on 5 August 2020).

19. Pollán, M.; Pérez-Gómez, B.; Pastor-Barriuso, R.; Oteo, J.; Hernán, M.A.; Pérez-Olmeda, M.; Eng, J.L.S.M.; Fernández-García, A.; Cruz, I.; de Larrea, N.F.; et al. Prevalence of SARS-CoV-2 in Spain (ENE-COVID): A nationwide, population-based seroepidemiological study. Lancet 2020, 396, 535-544. [CrossRef]

20. Stringhini, S.; Wisniak, A.; Piumatti, G.; Azman, A.S.; Lauer, S.A.; Baysson, H.; De Ridder, D.; Petrovic, D.; Schrempft, S.; Marcus, K.; et al. Seroprevalence of anti-SARS-CoV-2 IgG antibodies in Geneva, Switzerland (SEROCoV-POP): A population-based study. Lancet 2020, 396, 313-319. [CrossRef]

21. Hippich, M.; Holthaus, L.; Assfalg, R.; Zapardiel-Gonzalo, J.; Kapfelsperger, H.; Heigermoser, M.; Haupt, F.; Ewald, D.A.; Welzhofer, T.C.; Marcus, B.A.; et al. A Public Health Antibody Screening Indicates a 6-Fold Higher SARS-CoV-2 Exposure Rate than Reported Cases in Children. Med 2021, 2, 149-163. [CrossRef] [PubMed]

22. Pastor-Barriuso, R.; Perez-Gomez, B.; Hernan, M.A.; Perez-Olmeda, M.; Yotti, R.; Oteo, J.; Sanmartín, J.L.; León-Gómez, I.; Fernández-García, A.; Fernández-Navarro, P.; et al. Infection fatality risk for SARS-CoV-2: A nationwide seroepidemiological study in the non-institutionalized population of Spain [Internet]. Epidemiology 2020. Available online: http://medrxiv.org/ lookup/doi/10.1101/2020.08.06.20169722 (accessed on 2 December 2020).

23. Perez-Saez, J.; Lauer, S.A.; Kaiser, L.; Regard, S.; Delaporte, E.; Guessous, I.; Stringhini, S.; Azman, A.S.; Serocov-POP Study Group. Serology-informed estimates of SARS-CoV-2 in-fection fatality risk in Geneva, Switzerland. Lancet Infect. Dis. 2021, 21, 69-70. [CrossRef]

24. McDade, T.W.; McNally, E.M.; Zelikovich, A.S.; D'Aquila, R.; Mustanski, B.; Miller, A.; Vaught, L.A.; Reiser, N.L.; Bogdanovic, E.; Fallon, K.S.; et al. High seroprevalence for SARS-CoV-2 among household members of essential workers detected using a dried blood spot assay. PLoS ONE 2020, 15, e0237833. [CrossRef]

25. WHO. Keep Health Workers Safe to Keep Patients Safe: WHO [Internet]. 2020. Available online: https://www.who.int/news/ item/17-09-2020-keep-health-workers-safe-to-keep-patients-safe-who (accessed on 28 March 2021). 
26. Yao, Y.; Wang, H.; Liu, Z. Expression of ACE2 in airways: Implication for COVID-19 risk and disease management in patients with chronic inflammatory respiratory diseases. Clin. Exp. Allergy 2020, 50, 1313-1324. [CrossRef] [PubMed]

27. Ibekwe, T.S.; Fasunla, A.J.; Orimadegun, A.E. Systematic Review and Meta-analysis of Smell and Taste Disorders in COVID-19. OTO Open 2020, 4. [CrossRef]

28. Tsang, T.K.; Lau, L.L.; Cauchemez, S.; Cowling, B.J. Household Transmission of Influenza Virus. Trends Microbiol. 2016, 24, 123-133. [CrossRef]

29. Laxminarayan, R.; Wahl, B.; Dudala, S.R.; Gopal, K.; Mohan B., C.; Neelima, S.; Reddy, K.S.J.; Radhakrishnan, J.; Lewnard, J.A. Epidemiology and transmission dynamics of COVID-19 in two Indian states. Science 2020, 370, 691-697. [CrossRef] [PubMed]

30. Rach, S.; Günther, K.; Hadeler, B. Participants who were difficult to recruit at baseline are less likely to complete a follow-up questionnaire-Results from the German National Cohort. BMC Med. Res. Methodol. 2020, 20, 1-7. [CrossRef] [PubMed] 